\title{
Seroprevalence of anti-SARS-CoV-2 IgG antibodies in Kenyan blood donors
}

\begin{abstract}
Sophie Uyoga ${ }^{1 *}+$, Ifedayo M. O. Adetifa, ${ }^{1,2}+$, Henry K. Karanja ${ }^{1}+$, James Nyagwange $^{1}+$, James Tuju1, Perpetual Wanjiku${ }^{1}$, Rashid Aman $^{3}$, Mercy Mwangangi ${ }^{3}$, Patrick Amoth ${ }^{3}$, Kadondi Kasera ${ }^{3}$, Wangari Ng'ang'a ${ }^{4}$, Charles Rombo ${ }^{5}$, Christine Yegon ${ }^{5}$, Khamisi Kithi $^{5}$, Elizabeth Odhiambo $^{5}$, Thomas Rotich ${ }^{5}$, Irene Orgut ${ }^{5}$, Sammy Kihara ${ }^{5}$, Mark Otiende ${ }^{1}$, Christian Bottomley ${ }^{2}$, Zonia N. Mupe ${ }^{1}$, Eunice W. Kagucia ${ }^{1}$, Katherine E. Gallagher ${ }^{1,2}$, Anthony Etyang ${ }^{1}$, Shirine Voller, ${ }^{1,2}$, John N. Gitonga ${ }^{1}$, Daisy Mugo' ${ }^{1}$, Charles N. Agoti ${ }^{1}$, Edward Otieno ${ }^{1}$, Leonard Ndwiga $^{1}$, Teresa Lambe ${ }^{6}$, Daniel Wright $^{6}$, Edwine Barasa $^{1}$, Benjamin Tsofa', Philip Bejon ${ }^{1,6}$, Lynette I. Ochola-Oyier', Ambrose Agweyu' ${ }^{1}$, J. Anthony G. Scott ${ }^{1,2}$, George M. Warimwe ${ }^{1,6}$ *

${ }^{1}$ EMRI-Wellcome Trust Research Programme, Kilifi, Kenya. ${ }^{2}$ Department of Infectious Diseases Epidemiology, London School of Hygiene and Tropical Medicine, Keppel Street, London, UK. ${ }^{3}$ Ministry of Health, Government of Kenya, Nairobi, Kenya. ${ }^{4}$ Presidential Policy and Strategy Unit, The Presidency, Government of Kenya. ${ }^{5}$ Kenya National Blood Transfusion Services, Ministry of Health, Nairobi, Kenya. ${ }^{6}$ Nuffield Department of Medicine, Oxford University, UK.
\end{abstract}

*Corresponding author. Email: suyoga@kemri-wellcome.org

†These authors contributed equally to this work.

$\ddagger$ These authors contributed equally to this work.

The spread of SARS-CoV-2 in Africa is poorly described. The first case of SARS-CoV-2 in Kenya was reported on March 12, 2020 and an overwhelming number of cases and deaths were expected but by July 31,2020 there were only 20,636 cases and 341 deaths. However, the extent of SARS-CoV-2 exposure in the community remains unknown. We determined the prevalence of anti-SARS-CoV-2 IgG among blood donors in Kenya in April-June 2020. Crude seroprevalence was 5.6\% (174/3098). Population-weighted, testperformance-adjusted national seroprevalence was $4.3 \%(95 \% \mathrm{CI} 2.9-5.8 \%)$ and was highest in urban counties, Mombasa (8.0\%), Nairobi (7.3\%) and Kisumu (5.5\%). SARS-CoV-2 exposure is more extensive than indicated by case-based surveillance and these results will help guide the pandemic response in Kenya, and across Africa.

Africa accounts for $17 \%$ of the global population (1) but by late July 2020 accounted for only $5 \%$ of the global COVID-19 cases and $3 \%$ of global COVID-19 deaths reported (2). This disparity has been attributed to limited capacity for diagnosis, timely implementation of stringent containment measures, a younger population structure and a predominance of asymptomatic and mild infections $(3,4)$. The first case of COVID-19 in Kenya was detected on March 12, 2020. Within one week the government instituted containment measures to limit the spread of the virus (5). By July 31, national surveillance recorded 20,636 cases and 341 deaths (6). This increase in cases is notably slower than the epidemic in Wuhan, Europe or the USA. Recently, it has been suggested that "the virus is spreading... ....with an attenuated outcome in Africa" but there are few data available to confirm or refute this assertion (7).

In countries affected early in the pandemic, serological surveillance was used to define cumulative incidence. For example, at the release of lockdown in Wuhan, 9.6\% of staff resuming work were found to have anti-SARS-CoV-2 antibodies (8). At the end of the epidemic wave in Spain, seropositivity was $5.0 \%$ in a random population sample of 60,897 (9). As the epidemic curve declined in Geneva, seroprevalence rose over three weeks from $4.8 \%$ to $10.9 \%$ (10). Currently, there are few estimates of SARS-CoV-2 seroprevalence in Africa in the literature (11).

Movement restrictions, in response to COVID-19, have limited the conduct of fieldwork for population-based serosurveys. Several countries have monitored seroprevalence in blood transfusion donors $(12,13)$ or expectant mothers attending ante-natal clinics (14). Here we report the results of a pragmatic national serosurvey using residual blood samples from transfusion donors across Kenya and a highly sensitive and specific assay for anti-SARS-CoV-2 spike immunoglobulin G (IgG).

We validated a widely-used enzyme linked-immunosorbent assay for SARS-CoV-2 IgG (15) with 910 serum samples from the pre-pandemic period and 174 sera from polymerase chain reaction (PCR) defined SARS-CoV-2 cases, and a well-characterized 5 sera panel from the National Institute of Biological Standards and Control (NIBSC) in the UK. For either receptor-binding domain (RBD) or whole spike, specificity was higher when using a ratio of the sample optical density (OD)/negative control OD than when using the raw sample OD plus 3 standard deviations to define seropositivity (table $\mathrm{S1}$ ). By using OD ratios, both $\mathrm{RBD}$ and spike 
ELISAs correctly classified 901 of 910 pre-pandemic samples as seronegative (table S1). However, the spike ELISA detected more seropositives (166 of 179 vs compared to 145 of 179 for RBD ELISA) among sera from SARS-CoV-2 PCR-positive individuals (fig. S2, A and B). Based on these data, we defined anti-SARS-CoV-2 IgG seropositivity as an OD ratio $>2$ and selected the spike ELISA for this study. The sensitivity and specificity, at this threshold, were $92.7 \%$ (95\% CI 87.9-96.1\%) and $99.0 \%$ (95\% CI 98.1-99.5\%), respectively (figs. S3, A and $\mathrm{B}$, S5, and S6; and table S1). As previously noted (15), the RBD and whole spike ELISA responses were highly correlated (fig. S3C), with very little inter-assay variation (fig. S4).

A total of 3,174 blood transfusion samples were collected from four Kenya National Blood Transfusion Service (KNBTS) regional blood transfusion centers that are supported by several satellites and hospitals between April 30 and June 16, 2020, from individuals aged 15-66 years. Approximately half of the samples were drawn in Mombasa; the remainder were evenly distributed between Nairobi, Kisumu and Eldoret (Fig. 1 and table S2). We excluded 18 duplicate samples, 56 records missing data on age or collection date and two records from individuals aged $\geq 65$ years. Policy in Kenya is to avoid blood donation from individuals $>65$ years, and we excluded these other data points as potentially unreliable. These exclusions left 3,098 samples for further analysis (Fig. 1).

Of the 3,098 samples, 174 were positive for anti-SARSCoV-2 Spike IgG giving a crude seroprevalence of $5.6 \%(95 \%$ CI 4.8-6.5\%). Crude seroprevalence varied by age $(P=0.046)$, ranging between 3.4-7.0\% among adults $15-54$ years; all 71 donors aged 55-64 years were seronegative (Table 1). Crude seroprevalence did not vary by sex $(P=0.50)$ but did vary geographically, from $1.9 \%$ in the Rift Valley region to $10.0 \%$ in the Western region $(P=0.002$, Table 1$)$.

Compared to the 2019 Kenya Population and Housing Census, our participants were more commonly male $(82.0 \%$ in our study vs $49.3 \%$ in the census), had more persons aged $25-34$ years (40.1\% vs $27.3 \%$ ) and more residents of coastal Counties ( $49.2 \%$ versus $9.1 \%$, Table 2 ). We therefore adjusted the prevalence estimate for the demographics of the sample using post-stratification, and for the sensitivity and specificity of the test.

The Bayesian population-weighted and test-adjusted seroprevalence for Kenya was 4.3\% (95\% CI 2.9-5.8\%, Table 1) and the posterior sensitivity and specificity estimates were $92.4 \%$ (95\% CI 88.0-95.6\%) and 98.9 (95\% CI 98.2-99.5\%), respectively. Seroprevalence was higher (4.2-5.2\%) in the younger age groups (15-44 years) and declined in the older age groups (45-64 years) but was similar for both sexes. Seroprevalence was highest for those living in Mombasa, Nairobi and the Western region, although the number of observations for the Western region was small. The directly standardized seroprevalence estimates are presented in table S3. Seroprevalence was also calculated for Counties that had at least 120 donors sampled. The three largest urban Counties of Mombasa, Nairobi, and Kisumu had SARS-CoV-2 seroprevalence of $8.0 \%$ (95\% CI $5.5-11.1 \%$ ), $7.3 \%$ (95\% CI $4.2-11.4 \%$ ) and $5.5 \%$ (95\% CI $2.8-9.6 \%$ ), respectively (table S4).

The frequency of blood donor sampling and crude seroprevalence estimates increased with time over the 7-week study period (Fig. 2). The median sample date was May 30, 2020 while the mid-point of the study was May 24, 2020. We did not adjust for sample date because the period of sampling varied for residents of different counties (Fig. 2C); instead we show the variation in crude prevalence over time (Fig. 2A).

Voluntary non-remunerated donors (VNRDs), who donate blood at community-based 'blood drives' comprised only $7.6 \%$ (236/3098) of our sample of donors; the remainder were family replacement donors (FRDs) who provide a unit of blood in compensation for a transfusion received by a sick relative. The two groups did not differ significantly by age $(P=0.15)$ or sex $(P=0.51$, table S5). Crude seroprevalence was $8.5 \%$ (20/236) for VNRDs and 5.4\% (154/2862) for FRDs. The median sample date for VNRDs (June 14, 2020) was two weeks later than that for FRDs (May 29, 2020).

Population exposure across Kenya, with a populationweighted test-adjusted seroprevalence of $4.3 \%$, is considerably higher than was previously thought, based on the cases and deaths reported to date. Seroprevalence was particularly high in the three urban counties; Mombasa (8.0\%), Nairobi (7.3\%) and Kisumu (5.5\%). Consistent with other studies, seroprevalence did not vary significantly by sex; $(9,10,16)$ however, it peaked in 35-44-year-olds and was lowest for those $\geq 45$ years, which is also consistent with existing reports where seroprevalence was found to be lower in older adults $(9,10)$.

SARS-CoV-2 seroprevalence in our study is comparable to estimates from large population-based serosurveys in China, Switzerland, Spain and the USA after the initial epidemic peak and following many tens of thousands of deaths $(9,10$, $17,18)$. Our results are also comparable to other surveys of blood donors in Brazil (13), Italy (12), and many parts of England (19). Kenya has an estimated population of 53 million in 2020 and $57 \%$ of the population is aged $15-64$ years. If the transfusion donor seroprevalence of $4.3 \%$ was applied to all 15-64-year-olds it would suggest approximately 1.3 million infections. However, by the median sample date, May 30, 2020, only 2093 cases had been detected (of which approximately $90 \%$ were asymptomatic) and 71 deaths among all ages (6). Although it is difficult to extrapolate our data directly to the whole population, they do strongly suggest that the infection is more widespread in Kenya than the current PCR test results suggest and indicate a need for more systematic testing. The current PCR testing strategy targets symptomatic 
individuals, health care workers, contacts of confirmed cases, international travelers, cross border truck drivers and residents of areas identified as hot spots."

What are the potential explanations for the divergence in the ratio of observed cases or deaths to serologically defined infections inferred from transfusion donors in Kenya, compared to many high-income countries? (i) The seroprevalence could be over-estimated because of bias in the selection or behavior of blood transfusion donors. (ii) Cases could be under-ascertained by national public health surveillance though it seems unlikely that reporting of deaths and severe cases could be reduced by several orders of magnitude, and hospitals in Kenya were not overwhelmed by admissions with respiratory illness. (iii) The steep demographic age-pyramid results in a smaller vulnerable age group. In Kenya, only 3.9\% of the population is aged 65 years or greater which is substantially less than, for example, $23.3 \%$ found in Italy; again, this would only explain a several-fold reduction in severe cases or deaths (4). (iv) There may be alternative mechanisms of immunity to SARS-CoV-2 including cell-mediated immunity $(20,21)$ perhaps as a result of $\mathrm{HCoV}$-elicited immunity $(22$, 23). Despite our prior work showing $\mathrm{HCoVs}$ circulate in Kenya (24), we did not identify evidence of cross-reactive antibodies to endemic coronaviruses in our validation study.

Although blood donors are not representative of the Kenyan population as a whole, we adjusted for demographic bias in the sample structure by standardization against the age, sex, and regional distribution of the Kenyan population. A substantial proportion (43\%) of the population of Kenya is outside the age-range (15-64 years) sampled in this study and the seroprevalence in children $<15$ years and adults $>65$ years is often lower $(9,10)$; our estimate for blood donors may be higher than the estimate for the population as a whole. Blood donors also differ from the general population in their risk of exposure to SARS-CoV-2. For instance, potential donors are excluded from giving blood if they have been ill during the last six months so the sample may underestimate the population prevalence of SARS-CoV-2 antibodies; on the other hand, people who are shielding at home are unlikely to be captured in our sample leading to an overestimate of seroprevalence. Our exploration of the two distinct populations of blood donors, FRDs and VNRDs, suggests variation in the seroprevalence by donor group but, of note, $92 \%(2862 / 3098)$ of our sample came from the group with lower seroprevalence and exclusion of VNRDs reduced the crude seroprevalence in our study little, from $5.6 \%$ to $5.4 \%$. Against these considerations, other countries have relied on blood transfusion donors for an early estimate of seroprevalence but later estimates from random population samples have not been substantially different $(25,26)$.

A key strength of this study is the rigorous validation that included testing positive and negative control samples from the target population, as well as reference plasma from the UK NIBSC as part of a WHO-coordinated effort on SARS-CoV2 seroepidemiology. In addition, we adopted a conservative seropositivity threshold to optimize assay specificity and sensitivity for our setting.

The pandemic response in countries with limited health care capacity has been driven by the aggressive implementation of control measures to limit transmission. Unfortunately, this strategy has been accompanied by enormous collateral costs, particularly in Africa. Modeled estimates of the disruptions of essential medical services, such as immunization and antenatal care, suggest an additional $\sim 253,500$ child deaths and 12,200 maternal deaths over six months in low and middle-income countries (27). In the absence of social protection, the economic effects of lockdown are debilitating so it is important to obtain an early measure of the trajectory of the epidemic.

Our study provides a national and regional estimate of population exposure to SARS-CoV-2 in an African country. The $4.3 \%$ prevalence in blood transfusion donors is in sharp contrast with the reported COVID-19 cases and deaths and supports the impression that disease may be attenuated in Africa (7).

\section{REFERENCES AND NOTES}

1. United Nations Department of Economic and Social Affairs Population Division, "World Urbanization Prospects: The 2018 Revision, custom data acquired via https://population.un.org/wup/DataQuery/," (2018).

2. Africa Centres for Diseases Control and Prevention, "Coronavirus Disease 2019 (COVID-19). https://africacdc.org/covid-19/. Accessed 21 July 2020," (2020).

3. B. Z. Diop, M. Ngom, C. Pougué Biyong, J. N. Pougué Biyong, The relatively young and rural population may limit the spread and severity of COVID-19 in Africa: A modelling study. BMJ Glob. Health 5, e002699 (2020). doi:10.1136/bmigh-2020002699 Medline

4. J. B. Dowd, L. Andriano, D. M. Brazel, V. Rotondi, P. Block, X. Ding, Y. Liu, M. C. Mills, Demographic science aids in understanding the spread and fatality rates of COVID-19. Proc. Natl. Acad. Sci. U.S.A. 117, 9696-9698 (2020). doi:10.1073/pnas.2004911117 Medline

5. Ministry of Health Kenya, "Press Statement on the update of the coronavirus in the country and response measures. https://www.health.go.ke/wpcontent/uploads/2020/03/Coronavirus-Press-Statement-March-17-2020.pdf," (2020).

6. Ministry of Health Kenya, "COVID-19 Situation Reports (SITREP). https://www.health.go.ke/\#1591180376422-52af4cle-256b" (2020).

7. M. Mbow, B. Lell, S. P. Jochems, B. Cisse, S. Mboup, B. G. Dewals, A. Jaye, A. Dieye, M. Yazdanbakhsh, COVID-19 in Africa: Dampening the storm? Science 369, 624626 (2020). doi:10.1126/science.abd3902 Medline

8. X. Wu, B. Fu, L. Chen, Y. Feng, Serological tests facilitate identification of asymptomatic SARS-CoV-2 infection in Wuhan, China. J. Med. Virol. 92, 17951796 (2020). doi:10.1002/imv.25904 Medline

9. M. Pollán, B. Pérez-Gómez, R. Pastor-Barriuso, J. Oteo, M. A. Hernán, M. PérezOlmeda, J. L. Sanmartín, A. Fernández-García, I. Cruz, N. Fernández de Larrea, M. Molina, F. Rodríguez-Cabrera, M. Martín, P. Merino-Amador, J. León Paniagua, J. F. Muñoz-Montalvo, F. Blanco, R. Yotti; ENE-COVID Study Group, Prevalence of SARS-COV-2 in Spain (ENE-COVID): A nationwide, population-based seroepidemiological study. Lancet 396, 535-544 (2020). doi:10.1016/S01406736(20)31483-5 Medline 
10. S. Stringhini, A. Wisniak, G. Piumatti, A. S. Azman, S. A. Lauer, H. Baysson, D. De Ridder, D. Petrovic, S. Schrempft, K. Marcus, S. Yerly, I. Arm Vernez, O. Keiser, S. Hurst, K. M. Posfay-Barbe, D. Trono, D. Pittet, L. Gétaz, F. Chappuis, I. Eckerle, N. Vuilleumier, B. Meyer, A. Flahault, L. Kaiser, I. Guessous, Seroprevalence of antiSARS-CoV-2 lgG antibodies in Geneva, Switzerland (SEROCoV-POP): A population-based study. Lancet 396, 313-319 (2020). doi:10.1016/S01406736(20)31304-0 Medline

11. M. G. Chibwana, K. C. Jere, R. Kamng'ona, J. Mandolo, V. Katunga-Phiri, D. Tembo, N. Mitole, S. Musasa, S. Sichone, A. Lakudzala, L. Sibale, P. Matambo, I. Kadwala, R. L. Byrne, A. Mbewe, B. Morton, C. Phiri, J. Mallewa, H. C. Mwandumba, E. R. Adams, S. B. Gordon, K. C. Jambo, High SARS-CoV-2 seroprevalence in Health Care Workers but relatively low numbers of deaths in urban Malawi. Wellcome Open Res. 5, 199 (2020). doi:10.12688/wellcomeopenres.16188.1 Medline

12. L. Valentiet al., SARS-CoV-2 seroprevalence trends in healthy blood donors during the COVID-19 Milan outbreak. medRxiv 2020.2005.2011.20098442 [Preprint] 31 May 2020. https://doi.org/10.1101/2020.05.11.20098442.

13. L. Amorim Filho, C. L. Szwarcwald, S. O. G. Mateos, A. C. M. P. Leon, R. A. Medronho, V. G. Veloso, J. I. F. Lopes, L. C. M. S. Porto, A. Chieppe, G. L. Werneck; Grupo Hemorio de Pesquisa em Covid-19. Seroprevalence of anti-SARS-CoV-2 among blood donors in Rio de Janeiro, Brazil. Rev. Saude Publica 54, 69 (2020). 10.11606/s1518-8787.2020054002643 Medline

14. D. D. Flannery, S. Gouma, M. B. Dhudasia, S. Mukhopadhyay, M. R. Pfeifer, E. C. Woodford, J. S. Gerber, C. P. Arevalo, M. J. Bolton, M. E. Weirick, E. C. Goodwin, E. M. Anderson, A. R. Greenplate, J. Kim, N. Han, A. Pattekar, J. Dougherty, 0. Kuthuru, D. Mathew, A. E. Baxter, L. A. Vella, J. Weaver, A. Verma, R. Leite, J. S. Morris, D. J. Rader, M. A. Elovitz, E. J. Wherry, K. M. Puopolo, S. E. Hensley, SARSCoV-2 seroprevalence among parturient women in Philadelphia. Sci. Immunol. 5 , eabd5709 (2020). doi:10.1126/sciimmunol.abd5709 Medline

15. F. Amanat, D. Stadlbauer, S. Strohmeier, T. H. O. Nguyen, V. Chromikova, M. McMahon, K. Jiang, G. A. Arunkumar, D. Jurczyszak, J. Polanco, M. BermudezGonzalez, G. Kleiner, T. Aydillo, L. Miorin, D. S. Fierer, L. A. Lugo, E. M. Kojic, J. Stoever, S. T. H. Liu, C. Cunningham-Rundles, P. L. Felgner, T. Moran, A. GarcíaSastre, D. Caplivski, A. C. Cheng, K. Kedzierska, O. Vapalahti, J. M. Hepojoki, V. Simon, F. Krammer, A serological assay to detect SARS-CoV-2 seroconversion in humans. Nat. Med. 26, 1033-1036 (2020). doi:10.1038/s41591-020-0913-5 Medline

16. A. T. Huang, B. Garcia-Carreras, M. D. T. Hitchings, B. Yang, L. C. Katzelnick, S. M. Rattigan, B. A. Borgert, C. A. Moreno, B. D. Solomon, L. Trimmer-Smith, V. Etienne, I. Rodriguez-Barraquer, J. Lessler, H. Salje, D. S. Burke, A. Wesolowski, D. A. T. Cummings, A systematic review of antibody mediated immunity to coronaviruses: Kinetics, correlates of protection, and association with severity. Nat. Commun. 11, 4704 (2020). doi:10.1038/s41467-020-18450-4 Medline

17. X. Xu, J. Sun, S. Nie, H. Li, Y. Kong, M. Liang, J. Hou, X. Huang, D. Li, T. Ma, J. Peng, S. Gao, Y. Shao, H. Zhu, J. Y.-N. Lau, G. Wang, C. Xie, L. Jiang, A. Huang, Z. Yang, K. Zhang, F. F. Hou, Seroprevalence of immunoglobulin $M$ and $G$ antibodies against SARS-CoV-2 in China. Nat. Med. 26, 1193-1195 (2020). doi:10.1038/s41591-020-0949-6 Medline

18. F. P. Havers et al., Seroprevalence of Antibodies to SARS-CoV-2 in 10 Sites in the United States, March 23-May 12, 2020. JAMA Intern Med, 10.1001/jamainternmed.2020.4130 (2020).

19. Public Health England, "Sero-prevalence epidemiology, England. https://assets.publishing.service.gov.uk/government/uploads/system/upload s/attachment_data/file/899301/Weekly_COVID19_Surveillance_Report_week_ 28.pdf in Weekly Coronavirus Disease 2019 (COVID-19) Surveillance Report 2020 Week 28," (2020).

20. A. Grifoni, D. Weiskopf, S. I. Ramirez, J. Mateus, J. M. Dan, C. R. Moderbacher, S. A. Rawlings, A. Sutherland, L. Premkumar, R. S. Jadi, D. Marrama, A. M. de Silva, A. Frazier, A. F. Carlin, J. A. Greenbaum, B. Peters, F. Krammer, D. M. Smith, S. Crotty, A. Sette, Targets of T Cell Responses to SARS-CoV-2 Coronavirus in Humans with COVID-19 Disease and Unexposed Individuals. Cell 181, 14891501.e15 (2020). doi:10.1016/i.cell.2020.05.015 Medline
21. N. Le Bert, A. T. Tan, K. Kunasegaran, C. Y. L. Tham, M. Hafezi, A. Chia, M. H. Y Chng, M. Lin, N. Tan, M. Linster, W. N. Chia, M. I.-C. Chen, L.-F. Wang, E. E. Ooi, S. Kalimuddin, P. A. Tambyah, J. G.-H. Low, Y.-J. Tan, A. Bertoletti, SARS-CoV-2specific T cell immunity in cases of COVID-19 and SARS, and uninfected controls. Nature 584, 457-462 (2020). doi:10.1038/s41586-020-2550-z Medline

22. A. Sette, S. Crotty, Pre-existing immunity to SARS-CoV-2: The knowns and unknowns. Nat. Rev. Immunol. 20, 457-458 (2020). doi:10.1038/s41577-0200389-z Medline

23. K. W. Ng et al., Pre-existing and de novo humoral immunity to SARS-CoV-2 in humans. bioRxiv 2020.2005.2014.095414 [Preprint] 23 July 2020. https://doi.org/10.1101/2020.05.14.095414.

24. G. P. Otieno, N. Murunga, C. N. Agoti, K. E. Gallagher, J. O. Awori, D. J. Nokes, Surveillance of endemic human coronaviruses (HCoV-NL63, OC43 and 229E) associated with childhood pneumonia in Kilifi, Kenya. Wellcome Open Res. 5, 150 (2020). 10.12688/wellcomeopenres.16037.2 Medline

25. H. Ward et al., Antibody prevalence for SARS-CoV-2 following the peak of the pandemic in England: REACT2 study in 100,000 adults. medRxiv 2020.2008.2012.20173690v20173692 [Preprint] 21 August 2020. https://doi.org/10.1101/2020.08.12.20173690.

26. P. H. England, National COVID-19 surveillance report week 40 https://assets.publishing.service.gov.uk/government/uploads/system/upload s/attachment_data/file/923668/Weekly_COVID19_Surveillance_Report_week_ 40.pdf (2020)

27. T. Roberton, E. D. Carter, V. B. Chou, A. R. Stegmuller, B. D. Jackson, Y. Tam, T. Sawadogo-Lewis, N. Walker, Early estimates of the indirect effects of the COVID19 pandemic on maternal and child mortality in low-income and middle-income countries: A modelling study. Lancet Glob. Health 8, e901-e908 (2020). do: 10.1016/S2214-109x(20)30229-1 Medline

28. S. Uyoga et al., Replication Data for: Seroprevalence of anti-SARS-CoV-2 lgG antibodies in Kenyan blood donors. Harvard Database (2020); doi:10.7910/DVN/RENVC9.

29. K. Ministry of Health, "Policy Guidelines on Blood Transfusion in Kenya. The National Blood Transfusion Service of Kenya. https://nbtskenya.or.ke/wpcontent/uploads/2019/02/Policy-Guidelines-on-Blood-Transfusion-inKenya.pdf. Accessed 20 July 2020," (2001).

30. A. Gelman, J. Hill, Data Analysis Using Regression and Multilevel/Hierarchical Models. A. M. Alvarez, N. L. Beck, L. L. Wu, Eds., Analytical Methods for Social Research (Cambridge University Press, New York, United States, 2007).

31. A. Gelman, C. Carpenter, Bayesian analysis of tests with unknown specificity and sensitivity. Journal of the Royal Statistical Society C, Applied Statistics. 2020. In press. Applied Statistics. http://www.stat.columbia.edu/ gelman/research/unpublished/specificity.pdf (2020).

32. A. Gelman, Prior distributions for variance parameters in hierarchical models. Bayesian Anal. 1, 515-534 (2006). doi:10.1214/06-BA117A

\section{ACKNOWLEDGMENTS}

We thank Prof Florian Krammer for providing the plasmids used to generate the RBD, spike protein, and CR3022 mAb used in this work. Development of SARS CoV-2 reagents was partially supported by the NIAID Centres of Excellence for Influenza Research and Surveillance (CEIRS) contract HHSN272201400008C. The COVID-19 convalescent plasma panel (NIBSC 20/118) and research reagent for SARS-CoV-2 Ab (NIBSC 20/130) were obtained from the National Institute for Biological Standards and Control, UK. We thank the blood donors and KNBTS staff who supported this work. We also thank the WHO SOLIDARITY II network for sharing of protocols and for facilitating the development and distribution of control reagents. This paper has been published with the permission of the Director, Kenya Medical Research Institute. This study was approved by the Scientific and Ethics Review Unit (SERU) of the Kenya Medical Research Institute (Protocol SSC 3426). Before the blood draw, donors gave individual consent for the use of their samples for research. Ethical approval was obtained for collection, storage and further use for the sample sets used in the validation assays (SERU numbers: 1433, 3149, 3426). Funding: This project was funded by the Wellcome Trust (grant numbers 220991/Z/20/Z; 203077/Z/16/Z). Sophie Uyoga is funded by DELTAS Africa Initiative [DEL-15-003], Isabella Ochola-Oyier 
is funded by a Wellcome Trust Intermediate Fellowship (107568/Z/15/Z),

Ambrose Agweyu is funded by a DFID/MRC/NIHR/Wellcome Trust Joint Global Health Trials Award (MR/R006083/1), J. Anthony G. Scott is funded by a

Wellcome Trust Senior Research Fellowship (214320) and the NIHR Health

Protection Research Unit in Immunisation, Ifedayo Adetifa is funded by an United

Kingdom's Medical Research Council and Department For International

Development through a African Research Leader Fellowship (MR/S005293/1)

and by the NIHR-MPRU at UCL (grant 2268427 LSHTM). GMW is supported by a

fellowship from the Oak Foundation. Charles N. Agoti is funded by the DELTAS

Africa Initiative [DEL-15-003], and the Department for International

Development and Wellcome (220985/Z/20/Z). Author contributions:

Conceptualization and methodology: SU, IMOA, AA, JAGS, EW, KG, AE, SV RA,

MM, PA, KK WN and GMW. Investigation: SU, HKK, JN, JT, PW, CR, CY, KKI, EO,

TR, IO, SK, ZNM, JNG, DM, CN, EO, LN, LIO and GMW. Formal analysis: JAGS, MO and CB. Validation: TL, DW, HKK, JN, JT, LIO and GMW. Resources and funding acquisition: SU, TL, PB and GMW. Supervision: EB, BT and PB. Writing - Original draft preparation: SU, IMOA, AA, JAGS and GMW. Writing - Review and editing: all authors. Competing interests: RA, MM, KK and PA are from the Ministry of Health, Government of Kenya. All other authors declare no competing interests. Data and materials availability: De-identified data has been published on the Havard dataverse server (28). This work is licensed under a Creative Commons Attribution 4.0 International (CC BY 4.0) license, which permits unrestricted use, distribution, and reproduction in any medium, provided the original work is properly cited. To view a copy of this license, visit

https://creativecommons.org/licenses/by/4.0/. This license does not apply to figures/photos/artwork or other content included in the article that is credited to a third party; obtain authorization from the rights holder before using such material."

\section{SUPPLEMENTARY MATERIALS}

science.sciencemag.org/cgi/content/full/science.abe1916/DC1

Materials and Methods

Figs. S1 to S6

Tables S1 to S5

References (29-32)

MDAR Reproducibility Checklist

20 August 2020; accepted 6 November 2020

Published online 11 November 2020

10.1126/science.abe1916 


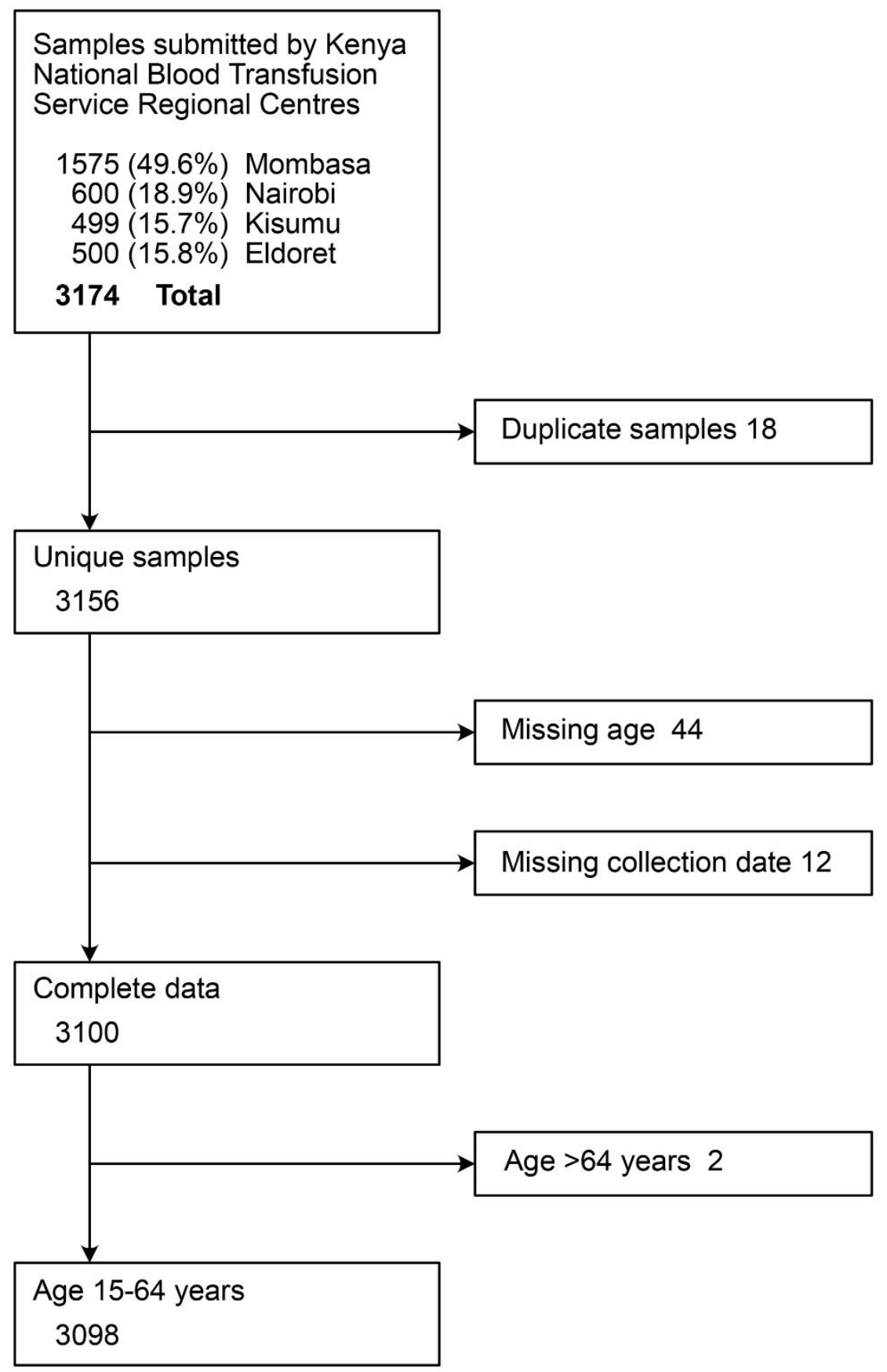

Fig. 1. Participant flow diagram for SARS-CoV-2 seroprevalence study of blood donors in Kenya. Exclusion criteria for the selection of samples with complete data. 
A

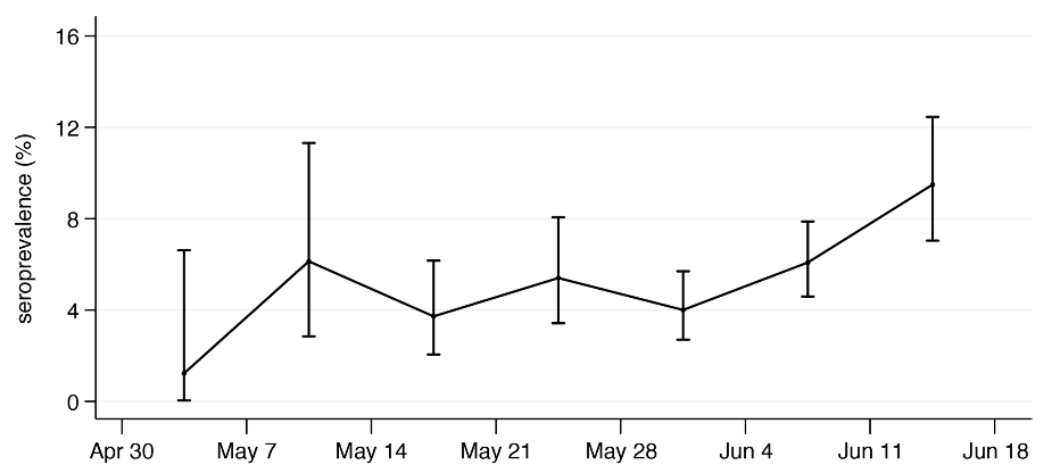

B
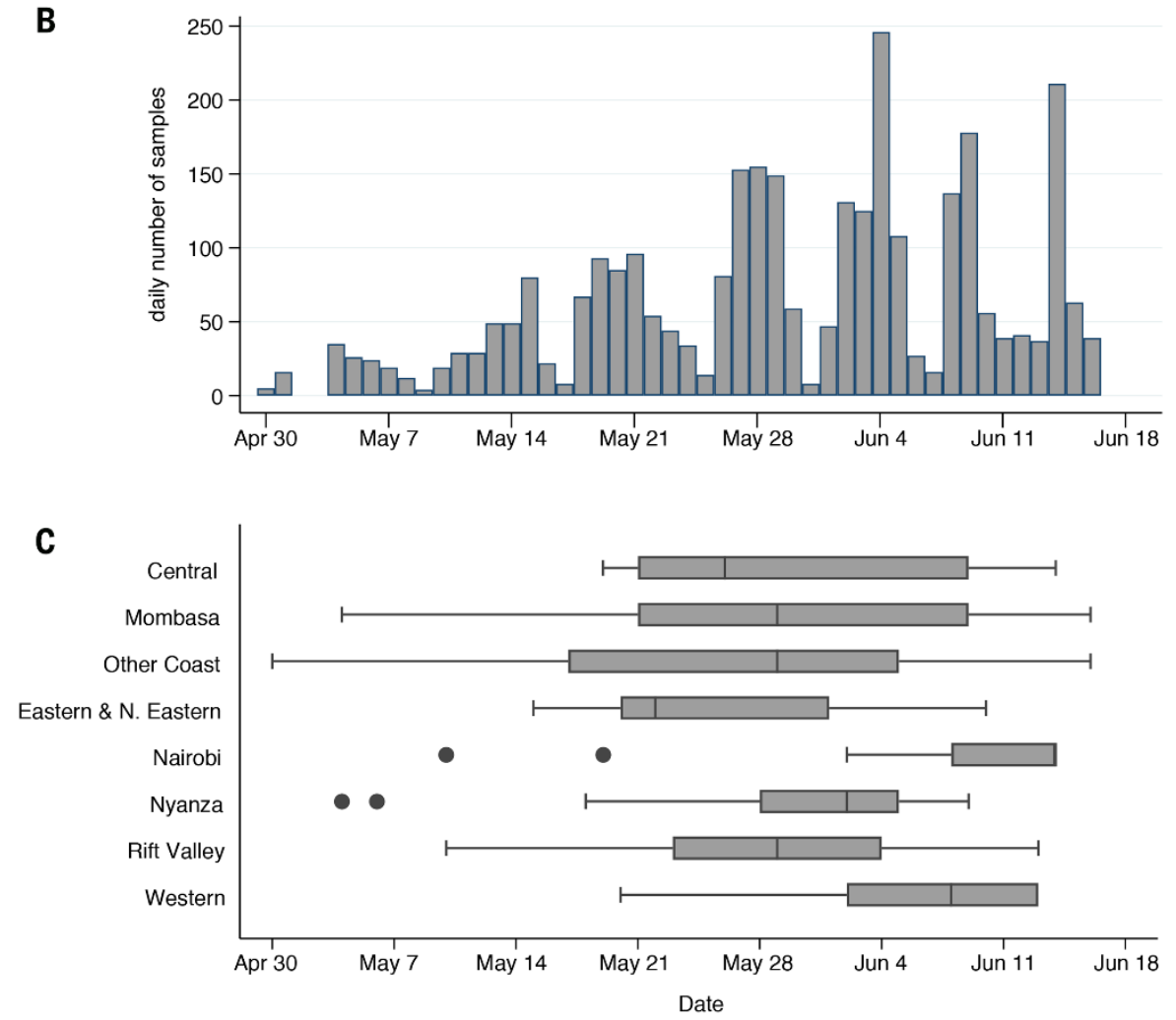

Fig. 2. Timeline of sampling for SARS-CoV-2 seroprevalence in blood donors in Kenya. Against the timeline of the sampling period, panel A shows the weekly crude seroprevalence and 95\% confidence interval, panel B shows the daily frequency of samples collected and panel $C$ shows the temporal distribution of samples by region. Proportion, counts and regional distribution of donors during the study period. 
Table 1. Crude, population-weighted, and test performance-adjusted SARS-CoV-2 anti-spike protein IgG seroprevalence by participant characteristics and regions. Prevalence estimates calculated using multilevel regression and post-stratification (MLRP) to account for differences in the sample population and the national population, subsequently adjusted for assay sensitivity and specificity.

\begin{tabular}{|c|c|c|c|c|c|c|c|c|c|}
\hline & \multirow[t]{2}{*}{ All samples } & \multirow[t]{2}{*}{$\begin{array}{l}\text { Seropositive } \\
\text { samples }\end{array}$} & \multicolumn{2}{|c|}{ Crude seroprevalence } & \multirow[t]{2}{*}{$\begin{array}{l}\text { Kenya popula- } \\
\text { tion }(2019 \text { Cen- } \\
\text { sus) }\end{array}$} & \multicolumn{2}{|c|}{$\begin{array}{l}\text { Bayesian population- } \\
\text { weighted seroprevalence* }\end{array}$} & \multicolumn{2}{|c|}{$\begin{array}{l}\text { Bayesian population- } \\
\text { weighted, test-adjusted se- } \\
\text { roprevalence* }\end{array}$} \\
\hline & & & $\%$ & $(95 \% \mathrm{CI})$ & & $\%$ & $(95 \% \mathrm{CI})$ & $\%$ & $(95 \% \mathrm{CI})$ \\
\hline \multicolumn{10}{|l|}{ Age } \\
\hline $15-24$ years & 808 & 49 & 6.1 & $4.5-7.9$ & $9,733,174$ & 5.1 & $3.7-6.9$ & 4.4 & $2.7-6.4$ \\
\hline $25-34$ years & 1242 & 66 & 5.3 & $4.1-6.7$ & $7,424,967$ & 4.9 & $3.6-6.4$ & 4.2 & $2.8-6.0$ \\
\hline $35-44$ years & 714 & 50 & 7.0 & $5.2-9.1$ & $4,909,191$ & 5.9 & $4.3-8.1$ & 5.2 & $3.3-7.7$ \\
\hline $45-54$ years & 263 & 9 & 3.4 & $1.6-6.4$ & $3,094,771$ & 3.8 & $1.9-6.0$ & 3.0 & $1.1-5.4$ \\
\hline $55-64$ years & 71 & 0 & 0 & & $1,988,062$ & 3.4 & $0.7-6.2$ & 2.9 & $0.7-5.7$ \\
\hline \multicolumn{10}{|l|}{ Sex } \\
\hline Male & 2540 & 146 & 5.7 & $4.9-6.7$ & $13,388,243$ & 4.4 & $2.9-6.2$ & 3.6 & $1.9-5.8$ \\
\hline Female & 558 & 28 & 5.0 & $3.4-7.2$ & $13,761,922$ & 5.5 & $4.4-6.8$ & 4.8 & $3.5-6.4$ \\
\hline \multicolumn{10}{|l|}{ Regions } \\
\hline Central & 105 & 7 & 6.7 & $2.7-13.2$ & $3,452,213$ & 5.6 & $2.9-10.0$ & 4.9 & $1.9-9.7$ \\
\hline Mombasa & 550 & 51 & 9.3 & $7.0-12.0$ & 792,072 & 8.3 & $6.1-10.9$ & 7.8 & $5.4-10.8$ \\
\hline Other Coast & 973 & 39 & 4.0 & $2.9-5.4$ & $1,671,097$ & 3.7 & $2.6-5.1$ & 2.9 & $1.6-4.6$ \\
\hline Eastern / N. Eastern & 242 & 11 & 4.5 & $2.3-8.0$ & $5,176,080$ & 4.3 & $2.5-7.0$ & 3.5 & $1.4-6.6$ \\
\hline Nairobi & 235 & 21 & 8.9 & $5.6-13.3$ & $3,002,314$ & 7.6 & $4.9-11.2$ & 7.1 & $4.2-11.2$ \\
\hline Nyanza & 442 & 30 & 6.8 & $4.6-9.5$ & $3,363,813$ & 6.0 & $4.2-8.4$ & 5.2 & $3.1-7.9$ \\
\hline Rift Valley & 481 & 8 & 1.7 & $0.7-3.3$ & $7,035,581$ & 2.1 & $1.1-3.6$ & 1.5 & $0.4-3.1$ \\
\hline Western & 70 & 7 & 10.0 & $4.1-19.5$ & $2,656,995$ & 7.0 & $3.5-13.1$ & 6.3 & $2.5-13.1$ \\
\hline Total & 3,098 & 174 & 5.6 & $4.8-6.5$ & $27,150,165$ & 4.9 & $3.9-6.2$ & 4.3 & $2.9-5.8$ \\
\hline
\end{tabular}

*Reweighted prevalence estimates based on demographic data from the 2019 Kenya Population and Housing Census. 
Table 2. General characteristics of the study population compared to the national population of Kenya. $\mathrm{N}$ is the number of individuals in each stratum.

\begin{tabular}{llllll}
\hline & & \multicolumn{2}{c}{ Blood transfusion samples } & \multicolumn{2}{c}{ Kenya National Census 2019 } \\
& & $\mathrm{N}$ & $\%$ & $\mathrm{~N}$ & $\%$ \\
\hline Age & 15-24 years & 808 & 26.1 & $9,733,174$ & 35.8 \\
& 25-34 years & 1,242 & 40.1 & $7,424,967$ & 27.3 \\
& 35-44 years & 714 & 23.0 & $4,909,191$ & 18.1 \\
& 45-54 years & 263 & 8.5 & $3,094,771$ & 11.4 \\
\multirow{3}{*}{ Sex } & 55-64 years & 71 & 2.3 & $1,988,062$ & 7.3 \\
\multirow{3}{*}{ Regions } & Male & 2540 & 82.0 & $13,388,243$ & 49.3 \\
& Female & 558 & 18.0 & $13,761,922$ & 50.7 \\
& Central & 105 & 3.4 & $3,452,213$ & 12.7 \\
& Mombasa & 550 & 17.8 & 792,072 & 2.9 \\
& Other Coast & 973 & 31.4 & $1,671,097$ & 6.2 \\
& Eastern / N. Eastern & 242 & 7.8 & $5,176,080$ & 19.1 \\
& Nairobi & 235 & 7.6 & $3,002,314$ & 11.1 \\
& Nyanza & 442 & 14.3 & $3,363,813$ & 12.4 \\
\multirow{2}{*}{ Total } & Rift Valley & 481 & 15.5 & $7,035,581$ & 25.9 \\
\hline
\end{tabular}

